\title{
Ultra low frequencies phenomena in Jovian decametric radio emission
}

\author{
O. V. Arkhypov ${ }^{1}$ and H. O. Rucker ${ }^{2}$ \\ 1 Institute of Radio Astronomy, National Academy of Sciences of Ukraine, Chervonopraporna 4, 61002 Kharkiv, Ukraine \\ e-mail: rai@ira.kharkov.ua \\ 2 Space Research Institute, Austrian Academy of Sciences, Schmidlstrasse 6, 8042 Graz, Austria \\ e-mail: rucker@oeaw.ac.at
}

Received 11 October 2005 / Accepted 24 January 2006

\section{ABSTRACT}

\begin{abstract}
System of periodic peaks is found in the power spectra of ULF flux oscillations of Jovian decametric emission, which is observed at fixed frequencies. This result could be interpreted in terms of an ionospheric Alfvén resonator near Jupiter. Standing Alfvén wave could explain the system of emission bands in the dynamic spectrum of decametric emission. The space period of such bands and the frequency of standing waves have been used to estimate electron number density in the low magnetosphere of Jupiter. The result is consistent with radio occultation data. Hence, the ULF modulation hypothesis appears validated.
\end{abstract}

Key words. planets and satellites: individual: Jupiter - magnetic fields - radiation mechanisms: non-thermal - plasmas - waves magnetohydrodynamics (MHD)

\section{Introduction}

It is widely accepted that waves of ultra low frequencies (ULF) in the Jovian magnetosphere can be studied only in situ, with space probes. Only electromagnetic, mainly decameter (DAM) radio emission is observable from the Earth. However, some theories connect the planetary radio emission with ULF Alfvén waves, which accelerate electrons and stimulate plasma instabilities with electromagnetic wave generation near Jupiter and other planets (Belcher 1987; Su et al. 2005; Zarka 1998). It has been supposed that Alfvén waves modulate the Jovian decametric emission (DAM) with the Io orbital period $(42.46 \mathrm{~h}$, Belcher 1987) and the wave bounce period ( 10 min, Bagenal \& Leblanc 1988).

Much higher frequencies $(\sim 20 \mathrm{~Hz})$ are predicted for DAM modulation in an ionospheric Alfvén resonator near Jupiter (Su et al. 2005). As near the Earth, this resonator could operate in vertical direction with shear Alfvén waves, which are trapped between the ionospheric boundary and the peak of the Alfvén phase speed. There are verifiable consequences for the resonator hypothesis: (a) the space modulation of DAM by a standing Alfvén wave; (b) multiple resonant eigenfrequencies in DAM flux variations. We search for these effects to verify the hypothesis of DAM Alfvén modulation and a Jovian resonator.

\section{S/NB-emission as detectors of ULF waves}

There are various theoretical mechanisms for DAM modulation by ULF Alfvénic waves. For example, Ergun et al. (2005) suggested that electron acceleration or modulation may provide the physical mechanism that transfers energy from the Alfvén wave to the S-burst. It has been supposed that the time interval between $S$-bursts reflects the period of the stimulating Alfvén wave (Su et al. 2005).
Another effect is the periodic inclination of the narrow radio beam by Alfvenic disturbance of a magnetic field (Arkhipov 2002). Zarka et al. (1997) argue that the average intensity profile of a S-burst is consistent with emission beaming in a widely open $\left(70^{\circ}-80^{\circ}\right)$ hollow cone of $\sim 2^{\circ}$ thickness, decreasing to zero at both (inner and outer) edges within $\sim 0.2^{\circ}$. The symmetry axis of such a radiation pattern is the magnetic vector at the position of the radio source. Hence, even $\sim 0.2^{\circ}$ variations of the magnetic direction could modulate the radio flux towards the Earth with this geometry.

The time-delay variations for Alfvénic displacements of the radio source in an inhomogeneous magnetic field are important (Arkhipov 2002). A typical S-burst (Fig. 1a) has a fixedfrequency duration of a few milliseconds and an instantaneous frequency band of a few tens of $\mathrm{kHz}$ (Ellis 1982). Although their origin is the subject of much debate, there are arguments for a small source dimension ( $10 \mathrm{~km}$, Ellis 1982; Zarka 1998). Moreover, the standard element of S-theories is the low group velocity of emission in the vicinity of its source for effective amplification of a radio wave by plasma instabilities (e.g. Melrose 1986; Zaitsev et al. 1986; Willes 2002). These properties could provide detectable effects by periodic displacements of radio sources by magnetospheric hydro-magnetic waves of very low frequencies. Thus time delay and frequency variations are possible.

S-bursts usually appear as lines in the dynamic spectrum, i.e. on the time-frequency plane. Such a form of the S-burst reflects the quasi-straight motion of a radio source along a magnetic line with a decrease of the emission frequency in accordance with the local gyrofrequency of electrons. However, there are S-bursts with a sinusoidal shape (Fig. 1a). In this case it is not possible to explain the drift rate using only the classical model based on the hypothesis of adiabatic motion of trapped electrons (Boudjada et al. 1997). The problem could be solved taking into consideration a parallel electric field (Galopeau et al. 1999) 

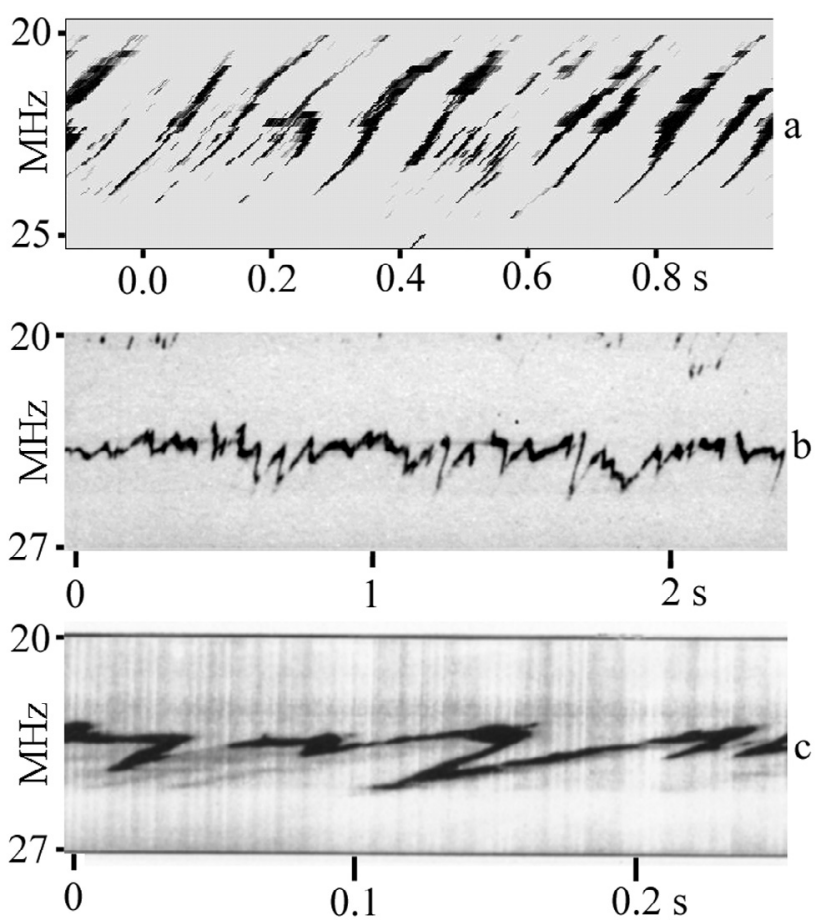

Fig. 1. a) The train of S-bursts with sinuousoidal shapes seen July 21, 1994 (Ryabov et al. 1997). b) The typical NB-event with oscillations seen November 12, 1988 (Riihimaa 1991). c) NB hooks (Riihimaa 1992) demonstrate the time-delay effect.

or an Alfvénic distortion of an active magnetic line (Arkhipov 2002). As Alfvén waves have parallel electric fields, these interpretations do not contradict each other. Parallel electric fields of Alfvén waves are considered as able to stimulate S-emission ( $\mathrm{Su}$ et al. 2005).

Sometimes S-bursts are associated with narrow band (NB) emission at quasi constant frequency (Boudjada et al. 2000). Apparently, in this case the radio source is fixed at some height above the planet. Therefore, it generates narrow band emission $(\sim 100 \mathrm{kHz})$ near the local gyrofrequency of electrons. In many cases such emission oscillates around the mean frequency in dynamic spectra (Fig. 1b). There is an obvious time-delay effect in NB-events: NB-hooks have seen, where the emission from one source is observed simultaneously at 2 to 3 different frequencies (Fig. 1c).

Such ambiguity of registration is known for S-bursts, too, as $\mathrm{f}, \mathrm{g}, \mathrm{h}, \mathrm{l}, \mathrm{n}, \mathrm{r}$ and $\mathrm{u}$ forms of J.J.Riihimaa's classification of S-spectra (Riihimaa 1991). The dispersion delay effects, the phase-bunching model have been proposed to explain this ambiguity (Willes 2002). However, the sinuous shapes of S/NB-events (Ryabov et al. 1997) can not be explained with this approach. Nevertheless, the wave-like forms of S/NB events are natural for harmonic displacements of a radio source (Arkhipov 2002). Alfvén waves are examples of such harmonic displacements in magnetized plasma.

Thus there are various reasons for the search for Alfvén modulation of DAM. Although the nature of the $\mathrm{S} / \mathrm{NB}$ source is still unknown, its emission could be used as a probe to study of ULF waves in the Jovian magnetosphere.

\section{Space modulation}

Particle acceleration, DAM generation or modulation are likely more effective in the antinodes (maxima) of the standing wave.

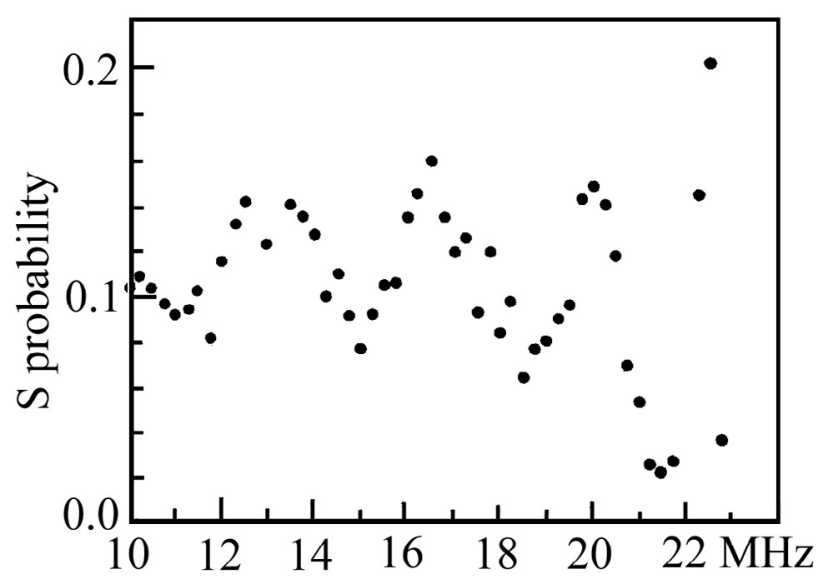

Fig. 2. The modulation of the mean probability of S-bursts in UTR-2 DAM spectra (Ryabov et al. 1985).

The DAM frequency decreases above Jupiter in accordance with the decrease in the local electron gyrofrequency $f_{\text {ce }}$. Hence, the ULF waves could be found in DAM dynamical spectra as quasi-periodic (in frequency) bands of radio emission. Indeed, it is well known that S/NB-emission in dynamic spectra is organized in bands near certain frequencies. The typical interval between such bands is 2-4 MHz (Riihimaa 1991; Ryabov et al. 1997).

Ryabov et al. (1985) found a quasi-periodic modulation of the mean probability of S-bursts in UTR-2 spectra (Fig. 2). This periodicity could be explained in terms of a standing wave. As its wavelength $\lambda$ is equal to the one of propagating waves with the same frequency $f_{w}$, we can estimate the phase speed of the ULF disturbance:

$V_{\mathrm{ph}}=\lambda f_{\mathrm{w}}$,

where $\lambda$ is the wavelength. This velocity is related to the electron number density for the Alfvén wave (e.g.: Nicholson 1983) as:

$V_{\mathrm{ph}}=\frac{1}{\sqrt{1 / V_{\mathrm{A}}^{2}+1 / c^{2}}}$

where $c$ is the speed of light; $V_{\mathrm{A}}=B /\left(4 \pi m_{\mathrm{i}} n_{\mathrm{i}}\right)^{1 / 2}$ is the Alfvén velocity. As a result, the electron density $N_{\mathrm{e}}$ in the radio source can be estimated for hydrogen plasma $\left(N_{\mathrm{e}}=n_{\mathrm{i}}\right)$.

There are the following frequencies of maximum/minimum probability of S-emission in Fig. 2: 11.75, 13, 15, 16.5, 18.5, 20, $21.4,22.5,22.8 \mathrm{MHz}$. We use the frequencies $f_{1}$ and $f_{2}$ of some neighboring maxima (or minima) to calculate the corresponding planetocentric radius-vectors $\overrightarrow{r_{1}}$ and $\overrightarrow{r_{2}}$ of radio sources, which are on the same Io activated magnetic line, with the VIP4 magnetic model (Connerney et al. 1998) and the standard $f=f_{\text {ce }}$ approximation. Then the half-wavelength of the standing wave is estimated as $\frac{\lambda}{2}=\left|\overrightarrow{r_{1}}-\overrightarrow{r_{2}}\right|$. The corresponding wavelengths $\lambda$ are shown in Table 1 . The estimations $N_{\mathrm{e}}$ are calculated with Eqs. (1), (2) and the most probable frequency of a S-burst recurrence of $f_{\mathrm{w}}=20 \mathrm{~Hz}$ (Carr \& Reyes 1999). The obtained $N_{\mathrm{e}}$ values are shown in Fig. 3 . To estimate the scale height $(H)$ correctly, the geometric altitude must be modified considering the spherical geometry and the influence of centrifugal force on co-rotating plasma. This modification is the special $Z$ altitude (Melrose 1967):

$Z=R_{\mathrm{J}}\left(1-R_{\mathrm{J}} / r\right)-\left(\Omega R_{\mathrm{J}}\right)^{2}\left(r^{3} / R_{\mathrm{J}}^{3}-1\right) /(2 L g)$, 
Table 1. Parameters of ULF waves.

\begin{tabular}{ccccccc}
\hline \hline $\begin{array}{c}f_{1} \\
{[\mathrm{MHz}]}\end{array}$ & $\begin{array}{c}f_{2} \\
{[\mathrm{MHz}]}\end{array}$ & $\begin{array}{c}\text { Hemi- } \\
\text { sphere }\end{array}$ & $\begin{array}{c}\lambda \\
{[\mathrm{km}]}\end{array}$ & $\begin{array}{c}V_{\mathrm{ph}} \\
{\left[\mathrm{km} \mathrm{s}^{-1}\right]}\end{array}$ & $\begin{array}{c}N_{\mathrm{e}} \\
{\left[\mathrm{cm}^{-3}\right]}\end{array}$ & $\begin{array}{c}Z-Z_{\mathrm{o}} \\
{[\mathrm{km}]}\end{array}$ \\
\hline 11.75 & 15 & $\mathrm{~N}$ & 13934 & 278684 & 148 & 11945 \\
& & $\mathrm{~S}$ & 13241 & 264811 & 264 & 12541 \\
13 & 16.5 & $\mathrm{~N}$ & 13138 & 262755 & 347 & 10295 \\
& & $\mathrm{~S}$ & 12466 & 249310 & 513 & 10945 \\
15 & 18.5 & $\mathrm{~N}$ & 11018 & 220363 & 1302 & 8053 \\
& & $\mathrm{~S}$ & 10449 & 208973 & 1619 & 8773 \\
16.5 & 20 & $\mathrm{~N}$ & 9772 & 195441 & 2505 & 6502 \\
& & $\mathrm{~S}$ & 9275 & 185508 & 2984 & 7269 \\
18.5 & 21.4 & $\mathrm{~N}$ & 7134 & 142686 & 7948 & 4830 \\
& & $\mathrm{~S}$ & 6779 & 135578 & 9054 & 5644 \\
20 & 22.5 & $\mathrm{~N}$ & 5623 & 112453 & 16615 & 3638 \\
& & $\mathrm{~S}$ & 5349 & 106972 & 18647 & 4480 \\
21.4 & 22.8 & $\mathrm{~N}$ & 2976 & 59525 & 75893 & 2873 \\
& & $\mathrm{~S}$ & 2835 & 56698 & 83968 & 3731 \\
\hline
\end{tabular}

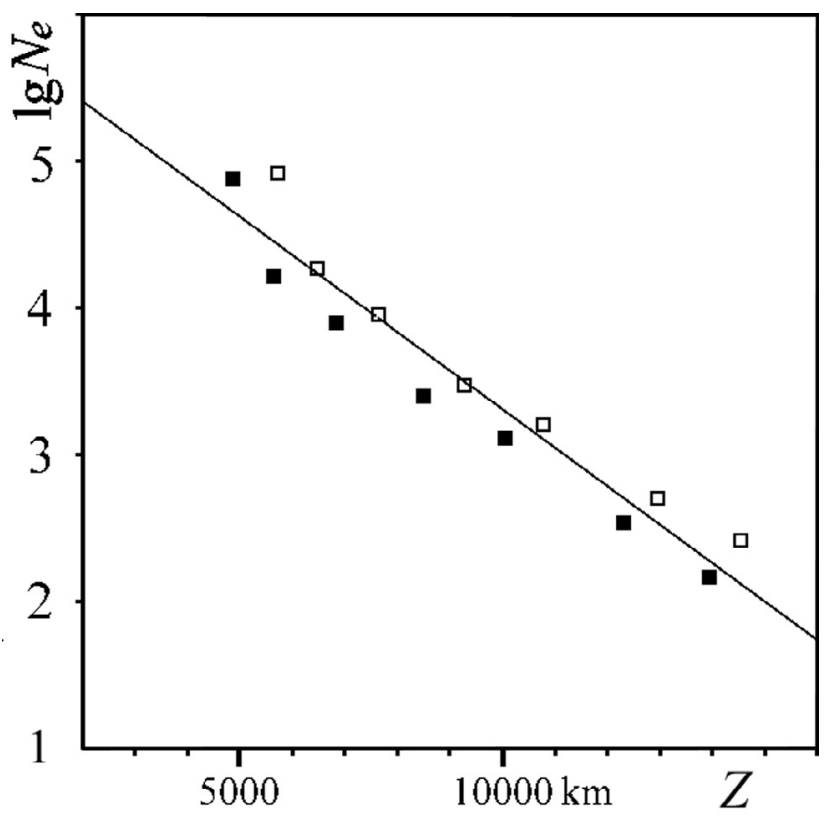

Fig. 3. Electron number density $N_{\mathrm{e}}\left(\mathrm{cm}^{-3}\right)$ at different altitudes $Z$ is estimated in terms of an Alfvén standing wave. The calculations are made for the northern (black squares) and southern (open squares) sources of Io-DAM with the VIP4 magnetic model, Io's Jovigraphical longitude of $260^{\circ}$ and a lead angle of $20^{\circ}$. The straight line approximation is found by a least squares fit using Eq. (4).

where: $R_{\mathrm{J}}=71372 \mathrm{~km}$ is the Jupiter radius; $r=\left(\left|\overrightarrow{r_{1}}\right|+\right.$ $\left.\left|\overrightarrow{r_{2}}\right|\right) / 2$ is the effective planetocentric distance; $\Omega=1.759 \times$ $10^{-4} \mathrm{rad} / \mathrm{s}$ is the angular velocity of Jupiter's rotation; $L=6$ is the McIlwain parameter for the Io torus; $g=25.9 \mathrm{~m} / \mathrm{s}^{2}$ is the acceleration of gravity in the Jovian exobase.

According to Melrose (1967), the electron density near Jupiter in the approximation of diffusion equilibrium can be described in the form:

$N_{\mathrm{e}}=N_{\mathrm{o}} \exp \left[-\left(Z-Z_{\mathrm{o}}\right) /(2 H)\right]$,

where $N_{\mathrm{o}}$ is the electron number density at height $Z_{\mathrm{o}} ; H=$ $k_{\mathrm{b}} T /\left(m_{\mathrm{i}} g\right)$ is the scale height $\left(k_{\mathrm{b}}\right.$ is the Boltzmann constant; $T$ is the temperature). Indeed, the obtained $\lg N_{\mathrm{e}}-Z$ relation (Fig. 3) satisfactorily corresponds to this equation. The optimal scale height $H=828 \pm 66 \mathrm{~km}$ and $\lg N_{\mathrm{o}}=5.41 \pm 0.16\left(\mathrm{~cm}^{-3}\right)$ near the ionospheric maximum $\left(Z_{\mathrm{o}}=2000 \mathrm{~km}\right)$ are close to the estimates from radio occultations $\left(400 \mathrm{~km}<H<960 \mathrm{~km} ; 4.2<\lg N_{\mathrm{o}}<\right.$ 5.6; Strobel \& Atreya 1983).
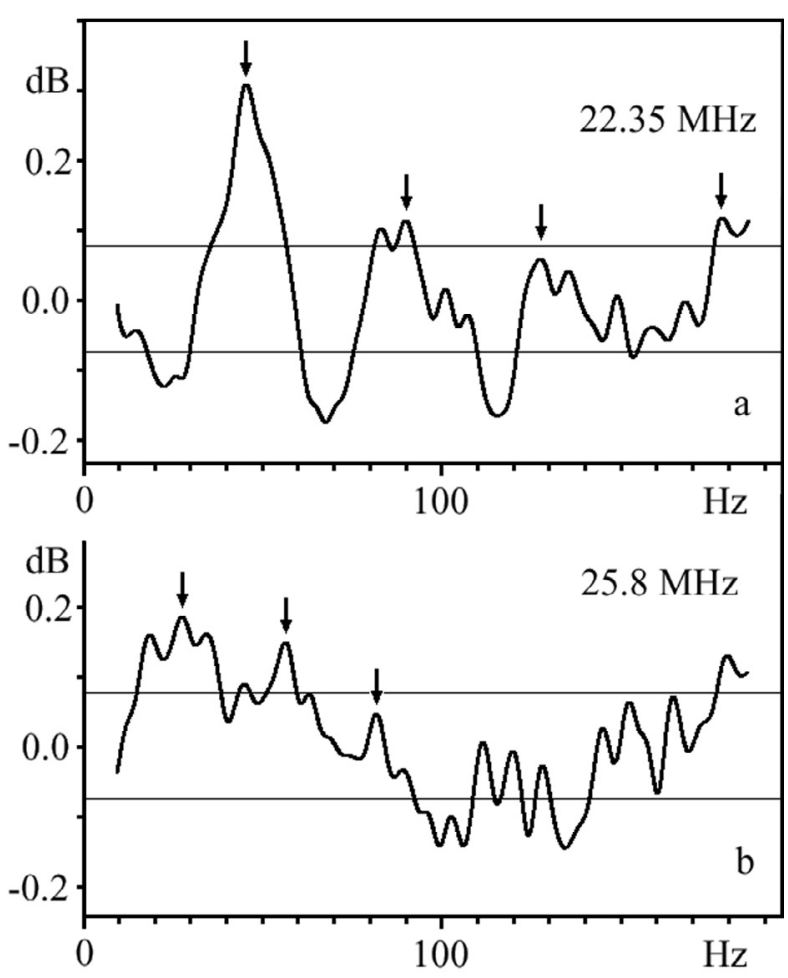

Fig. 4. a) The resonant peaks (arrowed) in the power spectrum of DAM flux variations at the fixed frequency $22.35 \mathrm{MHz}$ during the S/NB-event of March 30, 2000. b) Another S/NB-band at $25.8 \mathrm{MHz}$ simultaneously shows different resonances. The horizontal lines are the borders of the 0.98-significance interval.

\section{Time modulations of Jovian radio emission}

ULF properties of S/NB-emission have been rarely studied. Riihimaa (1991) found that the bursts in S-trains are repeated in rapid succession at quasi-periodic rates of 20-40 $\mathrm{s}^{-1}$. Carr $\&$ Reyes (1999) note that typical S-bursts are quasi-repetitive, recurring at rates between about 2 and $400 \mathrm{~Hz}$ over intervals of a few seconds, the most probable rate being $20 \mathrm{~Hz}$. At the frequencies below $20 \mathrm{~Hz}$, Calvert et al. (1988) investigated the random (Poisson) statistics for the cumulative percentage occurrence of S-burst spacing. However, their experimental points (Calvert et al. 1988, Fig. 5) show the systematic divergences from the Poisson approximation. Unfortunately, the significance of these spectral details is unknown.

To study S/NB periods in more detail, we used the dynamical spectrum of the S-storm of March 30, 2000, which was recorded by an observation compaign within the frame of the INTAS project 03-51-5727 with the UTR-2 radio telescope at 2 ms time resolution. The Fourier power spectrum $S(\omega)$ of the radio flux-time function at a fixed receiver frequency $f$ is calculated. It has been found that the relation between smoothed $\lg S(\omega)$ and the ULF frequency $\omega$ is quasi-linear with slightly positive curvature. Hence, this Fourier spectrum, smoothed with Bartlett spectral window, at $10 \mathrm{~Hz}<\omega<200 \mathrm{~Hz}$ can be approximated quadratically as $\lg S(\omega) \approx a+b \omega+c \omega^{2}$, where the constants $a, b$ and $c$ are found by the least squares method. To visualize the fine spectral details, the residuals $\lg (S(\omega))-\left(a+b \omega+c \omega^{2}\right)$ in $\mathrm{dB}$ are shown in Fig. 4.

The significance level is calculated for a standard $\chi^{2}$-distribution:

$F_{\chi 2}(x)=x^{\nu / 2-1}\left[2^{v / 2} \Gamma(v / 2)\right]^{-1} \exp (x / 2)$, 
Table 2. Comparison of found spectral peaks with predictions.

\begin{tabular}{ccccc}
\hline \hline Figure & $\begin{array}{c}\omega_{\text {obs }} \\
{[\mathrm{Hz}]}\end{array}$ & $\begin{array}{c}\omega_{\text {cal }}^{a} \\
{[\mathrm{~Hz}]}\end{array}$ & $\begin{array}{c}N_{\mathrm{i}}^{a} \\
{\left[\mathrm{~cm}^{-3}\right]}\end{array}$ & $\begin{array}{c}H^{a} \\
{[\mathrm{~km}]}\end{array}$ \\
\hline $2 \mathrm{a}$ & 45 & 51 & $2 \times 10^{4}$ & 780 \\
$2 \mathrm{a}$ & 90 & 89 & $2 \times 10^{4}$ & 780 \\
$2 \mathrm{~b}$ & 27 & 27.2 & $2 \times 10^{5}$ & 800 \\
$2 \mathrm{~b}$ & 56 & 50.2 & $2 \times 10^{5}$ & 800 \\
\hline
\end{tabular}

${ }^{a}$ Su et al. (2005), Fig. 7.

where $v=3 N \eta ; N$ is the number of processed realizations; $\eta=7$ is the ratio of the duration of one spectrum to the width of the Bartlett window.

The regular patterns of ULF spectral maxima are seen in Fig. 4a. Another S/NB-band at $25.8 \mathrm{MHz}$ observed simultaneously shows different resonances (Fig. 4b). There is a concurrence between the observed significant frequencies $\omega_{\text {obs }}$ and the calculated eigenmodes $\omega_{\text {cal }}$ of the ionospheric resonator in some models of Su et al. 2005 (Table 2). Although there is a difference in the model ionospheric density, $N_{\mathrm{i}}$, the scale height $H$ is practically the same.

According to the direct measurements by space probes in the polar magnetosphere of the Earth, there are field-aligned regions (cavities) of very depleted plasma density about 10 times lower than in the surrounding medium. Moreover, remote sensing experiments argue for the existence of analogous cavities near Jupiter, Saturn, Uranus and Neptune (Zarka 1998). If two radio sources are in two separate magnetic tubes with different $N_{\mathrm{i}}$ and consequently $V_{\mathrm{ph}}$, they are modulated by these ionospheric Alfvén resonators with different frequencies, even that of the same eigenmode. Therefore, the magnetospheric cavities could explain the differences in modulation frequencies and $N_{\mathrm{i}}$ estimations in Table 2.

To estimate the frequency range of the ionospheric resonator, the power spectra are calculated for Riihimaa's NB events (Riihimaa 1991, spectra Nos. 4, 6, 8-10, 12, 14, 15, 17-23, 26, $27,29,30,39,40,43,44,48,49,59,61-64,70,73-76)$. Figure 5 shows the distribution of frequencies of the main spectral peaks, calculated for each dynamical NB spectrum. Apparently, the ionospheric resonator works mainly on the frequencies $5-45 \mathrm{~Hz}$.

\section{Conclusions}

The analysis of observational data shows that the best candidates for ULF modulation are the oscillations of Jovian narrow band emissions (S-bursts and NB-events). Such oscillations are multi-frequency with significant spectral peaks near the resonant frequencies of the ionospheric Alfvén resonator (eigenmodes of $5-45 \mathrm{~Hz}$ ). S-bursts are repeated in trains with a typical frequency of $20 \mathrm{~Hz}$. It is suggested that this dominating frequency reflects the period of the Alfvénic wave, which accelerate electrons and stimulates or modulates S-emission. The hypothesis of a ionospheric resonator predicts a standing wave that stimulates S-bursts in many spectral bands. These bands must be localized quasi-periodically at different frequencies according to the local electron gyrofrequencies in the maxima of the standing wave. Predicted bands are found as the periodic frequency dependence of the S-burst occurrence probability. The Alfvén velocity and electron number density $\left(N_{\mathrm{e}}\right)$ are estimated from the

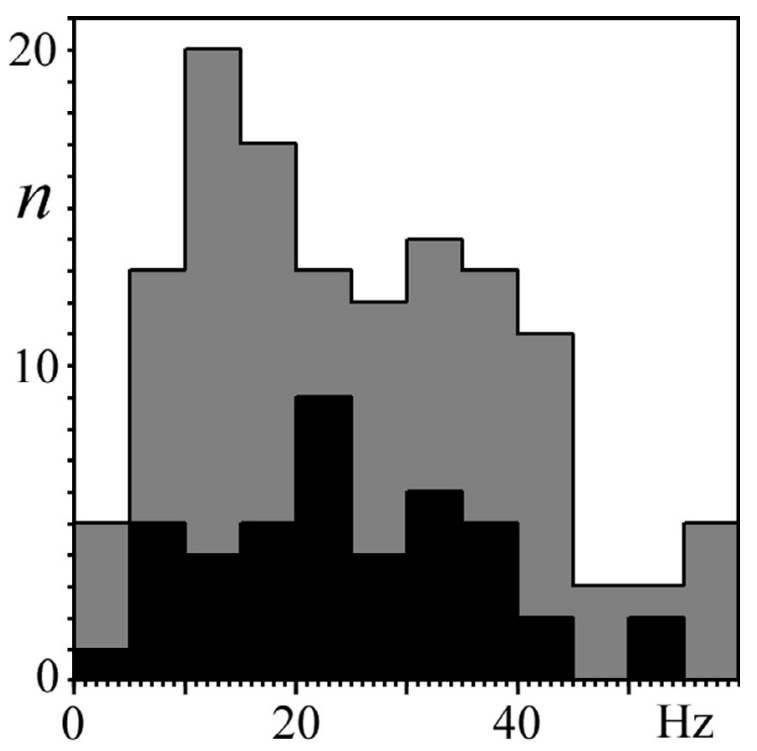

Fig. 5. Histogram of frequencies of the main spectral peak (black) and three maximum peaks (grey) in power spectra of NB-events.

wavelength and known period $(20 \mathrm{~Hz})$. The obtained $N_{\mathrm{e}}$ profile $\left(\lg N_{\mathrm{o}}\left[\mathrm{cm}^{-3}\right]=5.41 \pm 0.16 ; H=828 \pm 66 \mathrm{~km}\right)$ is consistent with radio occultation data. Hence, the obtained results are realistic, and the ULF modulation hypothesis appears valid.

Acknowledgements. We used the dynamical spectrum of the S-storm of March 30, 2000 , which was recorded by an observation compaign within the frame of the INTAS project 03-51-5727 (A. Lecacheux, H. R. Rucker, A. A. Konovalenko and Yu. Tokarev) with the UTR-2 radio telescope. We thank Dr. V. E. Shaposhnikov for fruitful discussions.

\section{References}

Arkhipov, A. V. 2002, Kinematika i Fizika Nebesnykh Tel, 18(5), 410 Bagenal, F., \& Leblanc, Y. 1988, A\&A, 197, 311 Belcher, J. W. 1987, Science, 238, 170

Boudjada, M. Y., Rucker, H. O., Galopeau, P. H. M., Kleewein, P., \& Mostetschnig, V. 1997, The contribution of the Riihimaa classification to the study of Jovian millisecond radio bursts, in Planetary Radio Emissions IV, ed. H. O. Rucker, S. J. Bauer, \& A. Lecacheux (Vienna: Austrian Academy of Sciences Press), 91

Boudjada, M. Y., Galopeau, P. H. M., Rucker, H. O., \& Lecacheux, A. 2000, A\&A, 363, 316 Calvert, W., Leblanc, Y., \& Ellis, G. R. A. 1988, ApJ, 335, 976

Carr, T. D., \& Reyes, F. 1999, J. Geohys. Res., 104, A11, 25127

Connerney, J. E. P., Acuna, M. H., Ness, N. F., \& Satoh, T. 1998, J. Geoph. Res., 103, A6, 11929

Ellis, G. R. A. 1982, Austr. J. Phys., 35, 2, 165

Ergun R. E., Su, Y.-J., Andersson, L., et al. 2005, Geophys. Res. Lett., in press

Galopeau, P. H. M., Boudjada, M. Y., \& Rucker, H. O. 1999, A\&A, 341, 918

Melrose, D. B. 1967, Planet. Space Sci., 15, 2, 381

Melrose, D. B. 1986, J. Geohys. Res., 91, A7, 7970

Nicholson, D. R. 1983, Introduction to plasma Theory (N.Y.: J. Wiley \& Sons), 163

Riihimaa, J. J. 1991, Earth, Moon and Planets, 53, 2, 157

Riihimaa, J. J. 1992, Wide-range high-resolution S-burst spectra of Jupiter (Oulu: Univ. of Oulu), 24

Ryabov, B. P., Arkhipov, A. V., \& Shevchenko, V. A. 1985, Astron. Vestn., 19, 4, 296

Ryabov, B. P., Zarka, P., Rucker, H. O., Ryabov, V. B., \& Boudjada, M. Y. 1997, Recurrent fine structures in Jovian S-burst emission, in Planetary Radio Emissions IV, ed. H. O. Rucker, S. J. Bauer, \& A. Lecacheux (Vienna: Austrian Academy of Sciences Press), 65

Strobel, D. F., \& Atreya, S. K. 1983, Ionosphere, in Physics of the Jovian magnetosphere, ed. A. J. Dessler (Cambridge: Cambridge University Press), 51, Fig. 2.4

Su, Y.-J., Jones, S. T., Ergun, R. E., et al. 2005, J. Geohys. Res., in press

Willes, A. J. 2002, J. Geohys. Res., 107, A5

Zaitsev, V. V., Zlotnik, E. Y., \& Shaposhnikov, V. E. 1986, A\&A, 169, 345

Zarka, P. 1998, J. Geohys. Res., 103, E9, 20159

Zarka, P., Ryabov, B. P., Ryabov, V. B., et al. 1997, On the origin of Jovian decameter radio bursts, in Planetary Radio Emissions IV, ed. H. O. Rucker, S. J. Bauer, \& A. Lecacheux (Vienna: Austrian Academy of Sciences Press), 51 